\title{
Self-adaptive Differential Particle Swarm using a Ring Topology for Multimodal Optimization
}

\author{
Gonzalo Nápoles $^{1}$, Isel Grau ${ }^{1}$, Rafael Bello ${ }^{1}$, Rafael Falcon ${ }^{2,3}$ and Ajith Abraham ${ }^{4,5}$ \\ ${ }^{1}$ Department of Computer Science, Universidad Central "Marta Abreu" de Las Villas, Santa Clara, Cuba \\ ${ }^{2}$ Research \& Engineering, Larus Technologies Corporation, Ottawa, Canada \\ ${ }^{3}$ Electrical Engineering and Computer Science, University of Ottawa, Ottawa, Canada \\ ${ }^{4}$ IT4Innovations, VSB-Technical University of Ostrava, Czech Republic \\ ${ }^{5}$ Machine Intelligence Research Labs (MIR Labs), USA \\ E-mail: gnapoles@uclv.edu.cu; igrau@uclv.edu.cu; rbellop@uclv.edu.cu; \\ rfalcon@uottawa.ca; ajith.abraham@ieee.org
}

\begin{abstract}
During the last couple of decades, evolutionary and swarm intelligence algorithms have significantly advanced the state of the art for both discrete and numerical optimization. Without niching strategies, they usually converge to a single optimum, even in multimodal search spaces where numerous global or local solutions exist. In the literature, several niching approaches have been proposed for simultaneously computing multiple optima, though most of them require some userspecified parameters that should be calculated a priori, i.e. additional knowledge about the problem domain is required. Recently, it was demonstrated that particle swarm optimization (PSO) using a ring topology for neighborhood definition can give rise to robust and parameterless niching methods. Nevertheless, their performance dramatically worsens when the dimensionality of the solution space hikes, thus increasing the number of local optima. This paper aims at enhancing the performance of these types of PSO-based algorithms by introducing two procedures: (1) a differential operator for improving the search ability and (2) a heuristic clearing operator for controlling the swarm diversity. Such operators are probabilistically activated through a novel self-adaptive learning strategy. Empirical results confirm the superiority of our proposed scheme with respect to six other competitive niching techniques.
\end{abstract}

Keywords-multimodal optimization; particle swarm optimizer; ring topology; differential operator; heuristic clearing.

\section{INTRODUCTION}

Evolutionary and swarm intelligence algorithms are stochastic search methods mimicking biological processes observed in nature. In contrast to the simple trajectory-based approaches, population-based techniques involve a set of artificial individuals that are capable of solving challenging optimization problems. The solution encoded by each agent is mapped onto a fitness value, which is used by the algorithm to craft its corporate search strategy, thus ignoring analytical properties of the heuristic cost function such as convexity, continuity, differentiability or gradient information. Despite their overwhelming success, these bio-inspired methods tend to converge to a single optimum, hence causing a gradual loss in population diversity as the search goes on [1].
In practice, most scientific and engineering optimization problems are highly multimodal, i.e. multiple global optima exist; therefore, it is often desirable to locate either all or a substantial number of these solutions [2] [3]. For example, industrial designers usually prefer to have a wide range of solutions to choose from, instead of only ending up with the best one according to the objective function selected. Actually, the final design is often decided not only considering the optimization results but introducing further factors like cost, simplicity and reliability [4].

In order to retain many local or global solutions found when optimizing multimodal functions, niching methods were introduced as a suitable extension of evolutionary algorithms [5]. Like a plethora of other computational models, niching mechanisms have drawn much inspiration from living organisms, where biological ecosystems are composed of several natural species exhibiting diverse features, which allow the development of numerous types of wildlife. It is assumed that a species is formed by individuals with similar biological traits that are capable of breeding among themselves yet are unable to do so with members of other species [6]. Thus, since niche resources are frequently finite, the density of the species heavily depends on both the amount of resources in the environment and the ability of the species members to exploit them. The fitness of an individual, then, measures its ability to exploit environmental resources to generate offspring [7].

Multimodal optimization methods, then, attempt to discover and maintain multiple subpopulations in a single run, where each niche corresponds to a specific peak of the fitness landscape. These methods have been developed to reduce the undesirable effects of genetic drift. Niching strategies should be capable to preserve the diversity in the artificial population, thus allowing individuals to simultaneously converge toward diverse solutions. On the other hand, niching methods are a convenient way to avoid potential stagnation or premature convergence in global optimization problems where many suboptimal solutions exist. It means that niching also provides an escaping alternative from local optima. 
Several niching methods have been proposed, including: fitness sharing, conserving techniques, restricted tournament selection, crowding methods, clearing procedures, clustering, species etc. [8]. Frequently, most of these algorithms require one of more parameters that enable the system to distinguish among different optima. Perhaps the most widely used is the niche radius, which defines the optimal separation distance between dissimilar niches. Setting this value requires a priori knowledge of the problem domain, which in many real-world problems is scant or simply unavailable [2] [3] [9].

Recently, X. Li proved that particle swarm optimization (PSO)-based schemes adopting a ring topology for local-best (lbest) neighborhood definition are capable of discovering multiple local/global optima and that they are robust and effective niching methods [10].In particular, one key advantage of such PSO niching algorithms is that there is no need to specify any niching parameter [10], which is an important contribution in multimodal optimization. However, these lbest models still exhibit some drawbacks that need to be circumvented in order to enhance their overall performance, such as: (a) they require a reasonably large population size uniformly distributed in the search space to form stable species across several local neighborhoods; (b) given that the local swarms in ring-topology-based PSO models may contain very few particles, their search capabilities are quite limited and (c) as a result of (a) and (b), when the dimensionality of the search space increases -and thus the number of local/global optima-, the percentage of the discovered optima dramatically drops.

To overcome these disadvantages, this paper makes the following contributions: (1) a hybrid extension of the ringtopology-based PSO algorithms is introduced, thus enhancing the performance of such models in presence of multimodality; (2) a novel differential operator that induces a controlled perturbation on the particles' trajectories is put forth, thus driving local swarms to unexplored regions of the search space and therefore increasing the likelihood of discovering more peaks; (3) a heuristic clearing procedure that is able to preserve the swarm diversity more efficiently without removing previously found solutions is devised and (4) an iteration-wise self-adaptive learning scheme that probabilistically activates both operators is depicted. As a result, we formalize a simple and powerful model called Self-adaptive differential PSO with heuristic clearing (S-PSO-DC) which considerably outperforms existing lbest niching strategies. Besides, the S-PSO-DC model does not require any additional knowledge about the problem domain, which makes it an appealing algorithm to deal with entangled multimodal problems.

The rest of the paper is organized as follows: In Section II, PSO basics are outlined and a brief overview of PSO models for multimodal optimization is provided. Section III dissects the proposed S-PSO-DC algorithm. The empirical analysis can be found in Section IV. As a final point, the authors provide observations and insights on further research in Section V.

\section{PARTICLE SWARM OPTIMIZATION}

PSO is an effective and robust global search method for solving challenging numerical optimization problems. This metaheuristic technique is concerned with a set of particles called a swarm which explores the search space trying to locate promising regions [11]. Therefore, particles are interpreted as solutions for the optimization problem and they are represented as points in an $n$-dimensional search space. In the case of standard PSO, each particle $X_{i}$ moves through the space using its own velocity $V_{i}$, a local memory of the best position $P_{i}$ it has ever obtained and the knowledge of the best solution $G_{i}$ found in its neighborhood. Equations (1) and (2) show how to update the particle's position based on these components, where $k$ indexes the current iteration, $0 \leq r_{1}, r_{2} \leq 1$ are random numbers drawn from a uniform distribution, whereas $c_{1}$ and $c_{2}$ are two positive user-specified parameters denoting the cognitive and the social aspects of the search, respectively. In addition, a novel factor called constriction coefficient $(\chi)$ was introduced by Clerc and Kennedy [12] to avoid the swarm explosion and ensure its convergence.

$$
\begin{gathered}
V_{i}^{(t+1)}=\chi\left(V_{i}^{(t)}+\mathrm{c}_{1} \mathrm{r}_{1}\left(P_{i}-X_{i}^{(t)}\right)+\mathrm{c}_{2} \mathrm{r}_{2}\left(G_{i}-X_{i}^{(t)}\right)\right) \\
X_{i}^{(t+1)}=X_{i}^{(t)}+V_{i}^{(t+1)}
\end{gathered}
$$

Topological neighborhoods are unrelated to the protocol in the particle's communication. They are grouped into two models: gbest (global topology) and lbest (local topology). In the first model, particles use global knowledge obtained from the entire swarm, which means that at each iteration, the best solution $G$ refers to the fittest particle in the whole population. In the case of lbest, each particle shares information with only a limited subset of the swarm members. Obviously, this feature induces a partition of the whole population into multiple subpopulations, each having its own topological global best. In other words, the lbest model involves not just a single global best record but several topological global best solutions $G_{r}$ emerge. Consequently, each particle adjusts its position in the search space using its own personal best information and also global knowledge about its fittest neighbor. In Section IV we showcase a novel lbest PSO algorithm to optimize multimodal functions that yielded a competitive performance and does not require any niching-related parameter specification.

A large number of multimodal methods using PSO update rules have been proposed, which are able to discover and maintain multiple optima in a single execution. As an illustration, the speciation-based PSO (SPSO) in [13] was motivated by the behavior of species-conserving approaches. Recently, other PSO models for solving multimodal problems were introduced [8]. Although these approaches are able to induce stable niching behavior, they frequently need to know the niche radius or other complex parameters should be estimated. In following Section III, we bring forward a novel lbest PSO algorithm for multimodal function optimization with competitive performance that does not require any nichingrelated parameter specification.

\section{SELF-ADAPTIVE DIFFERENTIAL PSO}

Recently, X. Li demonstrated in [10] that an lbest PSO using a ring topology is capable of inducing stable niching behavior in the swarm. The lbest ring model connects each particle with only two other particles, i.e. its immediate left and/or right neighbors, as opposed to the gbest model where every particle communicates with the very best particle in the 
entire swarm. Figure 1 illustrates the simplest form of this lbest topology called r2PSO, where a subpopulation is formed only by the current particle and its right neighbor. In a similar way, another model termed r3PSO may be also defined where each subpopulation is composed by three individuals: the current particle and its left and right neighbors. Given the limitations of these approaches described in Section I, our work, therefore, aims at improving r2PSO and r3PSO models.

It is easy to notice that each subpopulation may converge to a single optimum in the fitness landscape owing to the presence of different local attractors. Thus, niches naturally emerge from the global population without any knowledge of the problem domain. Yet since subpopulations involve only a few particles, the search capabilities of such models are quite poor, especially for large search spaces having both local and global optima. To overcome this disadvantage, we first introduce a differential operator to improve the search process in an lbest model, i.e. their exploration as well as exploitation abilities.
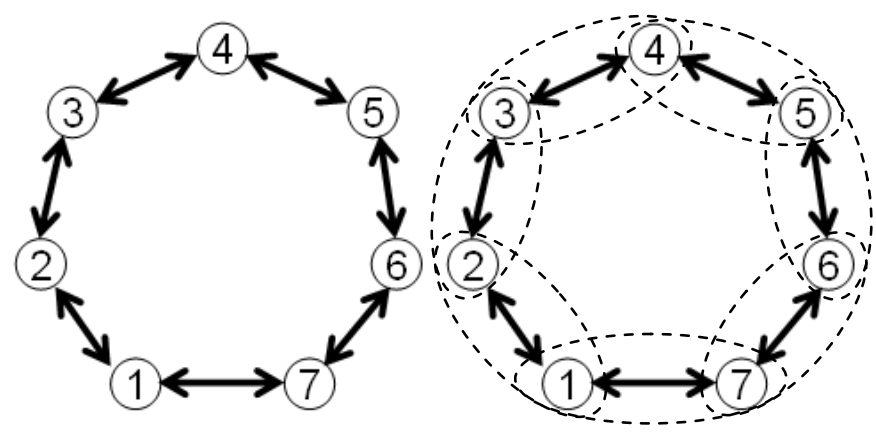

Figure 1. Simplest Local PSO using a Ring Topology a) Interaction among swarm particles and b) Overlapped subpopulations.

In addition, this paper presents a heuristic clearing operator for efficiently controlling the swarm diversity. It is well known that in multimodal fitness landscapes, peaks regularly have different size and height. In fact, in most cases, there exist very strong peaks attracting multiple agents, thus leading to the convergence of a significant portion of the global swarm to a single optimum. This problem could be reduced by using a reasonably large, uniformly distributed population yet at the expense of a steep computational price. To deal with this deficiency, we alternatively apply local heuristic clearing.

\section{A. Differential Operator}

When multimodal problems are tackled, the main advantage of the lbest model appears to lie in its slower convergence rate relative to the gbest model, for it allows concurrent discovery of several optima. Ironically, it is the slight interaction among the particles that is highly responsible for the meager performance of the PSO-based algorithms using a ring topology. To improve the search competence of such models, we introduce a simple differential operator that is straightforwardly inspired by the popular differential evolution strategy DE/current-to-rand/1 [14]. As a first step, we design a mutation operator as illustrated by equation (3), where $P_{i}$ denotes the personal best position of the current individual, $G_{r 1}$ and $G_{r 2}$ are the global best records achieved by two randomly selected particles and $0<F \leq 1$ is a real-valued parameter that regulates the differential amplification.

$$
\tilde{X}_{i}^{(t+1)}=P_{i}^{(t)}+F *\left(G_{r 1}^{(t)}-G_{r 2}^{(t)}\right)
$$

The mutated particle $\tilde{X}_{i}$ completely recombines the particle position based on the differential information by using the personal best memory $P_{i}$ as an attractor to guide the flying direction. Next, a selection operation takes place as depicted in equation (4) and assuming maximization, where $\tilde{X}_{i}$ is accepted as the current particle position only if it improves the local best position generated by the PSO update rule; otherwise, the mutated particle is rejected. Notice that the new differential operator introduces a perturbation based on the best global knowledge achieved by individuals belonging to neighboring subpopulations. Small values for the differential amplification parameter $F$ (e.g. $F<0.2)$ results in further exploitation around the personal best point, whereas large values (e.g. $F>$ 0.8 ) ensure higher exploration. To simplify the model, factor $F$ is fixed to 0.5 as it guarantees a good-enough tradeoff between exploitation and exploration.

$$
X_{i}^{(t+1)}= \begin{cases}\tilde{X}_{i}^{(t+1)}, & \text { if } f\left(\tilde{X}_{i}^{(t+1)}\right)>f\left(P_{i}^{(t+1)}\right) \\ X_{i}^{(t+1)}, & \text { otherwise }\end{cases}
$$

Generally speaking, Differential Evolution operators show an implicit self-adaptation capability which could improve the search in a ring-topology-based PSO. However, activating this operator at each iteration may significantly hit the algorithm's efficiency, as equation (4) demands that the objective function be additionally evaluated, which in a large number of realworld problems entails complex time-consuming operations. As a suitable alternative, we strongly suggest to trigger the proposed differential operator probabilistically. Nevertheless, a challenging issue remains: how to efficiently estimate the activation probability at each iteration? Section III-C sheds light on this important aspect.

\section{B. Heuristic Clearing Operator}

The basic clearing operator put forth by Petrowski [15] allocates the entire resources of a niche to a single individual: the dominant or species seed, while resets the fitness value of all the other individuals of the same subpopulation to zero. Then, to discover those agents belonging to the same niche, clearing uses the niche radius $\sigma$ : if the distance between the dominant $S_{i}$ and an agent $X_{l}$ is less than a fixed threshold $\sigma$, then $S_{i}$ and $X_{l}$ belong to same subpopulation. Towards the end, the global population is represented by several dominant individuals. In some sense, bio-inspired algorithms using a clearing procedure seem to be convenient to solve multimodal functions having large number of optima. Conversely, this mechanism is heavily sensitive to the niche radius.

Following a similar reasoning on the conventional clearing, we present a novel diversity-fostering procedure: heuristic clearing. This operator preserves the swarm diversity in lbest PSO schemes that employ a ring neighborhood topology yet needs not the specification of any niche parameter. Heuristic clearing only takes into account global optimal particles, as portrayed in (5). In this procedure the non-optimal particles are not considered since they could find better solutions, and thus they preserve their position in the search space. If the fitness value associated with the local best position $P_{i}$ achieved by the 
particle $X_{i}$ only differs in an infinitesimal constant $\varepsilon$ regarding the global best fitness value $F^{*}$, then the problem solution $P_{i}$ will be considered as a global optimum. In most real problems the value $F^{*}$ is often known by designers, but it also could be computed at each iteration as $F^{*}=\max _{\forall i}\left\{f\left(P_{i}\right)\right\}$, ensuring a further simplification of the clearing model.

$$
\left|f\left(P_{i}\right)-F^{*}\right|<\varepsilon
$$

Once the set of optimal particles $P$ is determined, then we sort particles in descending order of their personal fitness values. After that, a local clearing is applied. This procedure consists in reallocating the position $X_{k}$ of the $k$-th particle having the closest Euclidean distance from the current particle $P_{i}$, only if both particles $P_{i}$ and $P_{k}$ belong to the same niche. It allows preserving the swarm diversity and also the knowledge about highest discovered peaks. Although the proposed model seems to be quite reasonable, a significant problem persists: how to determine if two particles belong to the same niche? To address this question, we use the simplest form of the wellknown "hill valley" concept, i.e., two distinct particles $P_{i}$ and $P_{k}$ belong to the same niche $\Phi_{n}$ if their middle point $m$ is also an optimal solution. This idea is formalized in next equation (6):

$$
P_{i}, P_{k} \in \Phi_{n} \Leftrightarrow\left|f(m)-F^{*}\right|<\varepsilon \wedge P_{i} \neq P_{k}
$$

Lastly, it should be mentioned that this operator will be probabilistically activated during the algorithm execution, in order to reduce the extra computational cost needed to sort the swarm and compute the Euclidean distance. Assuming that the heuristic clearing operator is triggered at each iteration with probability 1.0 , then the temporal cost $O(N(P+\log N))$ will still be low for a given swarm size $N$.

\section{Self-adaptive learning scheme}

A large variety of real-world problems have irregular fitness landscapes where several local/global optima with different properties may exist. That is a serious problem which negatively affects both differential and clearing operators' performance. As a practical example, imagine a solution search space with numerous global optima of different size. At this time, local sub-swarms tend to quickly find global optima with higher size, which means that for these particles, the clearing probability should be high. But this design is unacceptable for local sub-swarms tending to form niches around peaks with lower size where particles need to exploit the neighborhood in detail. Then, based on the above illustration, it is easy to notice that probabilities used to activate both operators should be estimated according to the topological features of the particles in the different neighborhood (e.g. fitness values, distance to other particles, or progress in optimization).

The well-known no free lunch theorem [16] ensures that a single algorithm is unable to effectively solve all optimization problems regardless of the nature of the problem at hand. It confirms that there is a need to design more adaptive algorithms that boost the final performance of multimodal methods. For this reason, we devised a self-adaptive strategy for estimating the clearing probability $\left(p_{c}\right)$ during the search. Here we only focus on this parameter due to its influence on SPSO-DC's convergence. As to the $p_{d}$ factor, it will be anchored to 0.5 , which is a reasonable probability value.
The value of $p_{c}$ is computed by using a diversity measure ensuring the activation of the clearing procedure when the population is crowded. More specifically, this parameter is estimated as $p_{c}=1-\psi(S)$ where $\psi(\cdot) \in[0,1]$ is a function that computes the diversity over the swarm $S$ in the solution space. That is why the lower the swarm diversity, the higher the clearing probability. We adopted the average distance around each swarm center as $\psi(S)$, since Olorunda and Engelbrecht verified in [17] that this measure is less sensible to outliers, thus it is more robust with respect to other measures.

\section{PERFORMANCE STUDY}

In this section, we evaluate the ability of the proposed SPSO-DC scheme to handle multimodal problems with various characteristics. In other words, we are keen on studying the niching behavior of a revamped local PSO with a ring topology. To this end, the user needs to specify a level of accuracy $\varepsilon$ for each tested problem. If the absolute distance from a computed solution $f\left(P_{i}\right)$ to the known optimum value $F^{*}$ is less that the specified threshold $\varepsilon$, then $P_{i}$ is considered as a global peak. Our method is compared against six competitive niching models including: SPSO [13], FER-PSO [18] and also four lbest models using a ring topology which were widely commented in [10]. As a brief description, these approaches may be summarized as follows:

- r2PSO: lbest PSO with a ring topology, where each particle solely interacts with its immediate member on its left and right sides.

- r2hPSO: Idem as r2PSO but with no overlapping neighborhoods. Basically, multiple PSOs search in parallel like local hill climbers. This variant is more appropriate if the goal behind the optimization is to find global optima as well as local optima.

- r3PSO: lbest PSO with a ring topology, where each member only interacts with the particle to its right.

- r3hPSO: This model is similar to r3PSO, but with no overlapping neighborhoods, hence acting as multiple local hill climbers, being more suitable for finding global as well as local optima.

Both differential and heuristic clearing operators are integrated into the abovementioned lbest models, which lead to four new niching algorithms: r2PSO-DC, r3PSO-DC, r2hPSODC and r3hPSO-DC. It should be noticed that only SPSO requires the niche radius. Moreover, to gauge the general performance of all models, we lean on a method that identifies the species' dominants [13]. It checks if a niching algorithm has located all known global peaks. Hence, with exception of the SPSO algorithm, the radius factor is only needed to measure the performance of the models under consideration, which means it is not used in the optimization.

We also include two performance measures: the success rate and the peak ratio. The former is a ratio of the number of runs in which all global peaks were successfully located with respect to the total number of runs. The latter statistic measures the number of optimal solutions found in a run with regards to the total number of known global peaks. 


\section{A. Benchmark functions}

We tested 12 well-known problems taken from [10], which are commonly employed in niching literature since they exhibit different properties such as deceptiveness, multiple evenly and unevenly spaced global optima, solution spaces characterized by local/global peaks and high dimensionality. These functions may be categorized into four major groups: 1-D Deceptive Functions $\left(f_{1}, f_{2}, f_{3}\right), 1$-D Multimodal Functions $\left(f_{4}, f_{5}, f_{6}, f_{7}\right), 2$ D Multimodal Functions $\left(f_{8}, f_{9}, f_{10}\right)$, and also More Challenging Two or Higher-Dimensional Multimodal Functions $\left(f_{11}\right.$ and $\left.f_{12}\right)$ ranging from simpler to more intricate ones.

\section{B. Simulation results}

In all experiments discussed in this section we use the same parametric settings reported in [10]. It includes, for each tested function: a level of accuracy $\varepsilon$, the maximum number of evaluations $M$, the population size $N$ and the niche radius $r$. Notice again that the level of accuracy $\varepsilon$ and a fixed niche radius $r$ are exclusively for performance measurements. Table I shows the success rate averaged over 50 independent trials, for $f_{1}-f_{10}, f_{11}{ }^{2 \mathrm{D}}$, and $f_{12}{ }^{1 \mathrm{D}}$ which are relatively simple functions (although the search space of these functions is certainly tough for any niching algorithm, they have few global optima). From these simulations, a promising conclusion came out: r2PSODC, r3PSO-DC, r2hPSO-DC and r3hPSO-DC are able to find all solutions over 50 trials. As well, it is clear that lbest models, in general, show stable performance over SPSO and FER-PSO on all benchmarks under discussion.

Table II portrays the average peak ratios (over 50 runs) achieved by the algorithms in presence of more complicated functions. In this case, no algorithm was able to discover all peaks. Conversely, it is quite remarkable that improved lbest methods are much better equipped to handle these challenging functions. As an illustration, for problem $f_{11}{ }^{3 \mathrm{D}}$, r3PSO-DC reports a significant improvement with respect to its analogous method r3PSO; here the peak ratio increases by $31 \%$ which means that about 25 new optima were found. In general, r3PSO-DC seems to be more effective for optimizing search spaces having numerous global as well as local optima, while $\mathrm{r} 2 \mathrm{hPSO}-\mathrm{DC}$ is preferable for problems where only global peaks exist. Despite this, for search spaces having large number of optima (e.g. for problem $f_{11}^{4 \mathrm{D}}$ ), the algorithms' performance is still poor, even for the proposed models. This suggests the need to incorporate more competent optimizers in order to improve the scalability properties of lbest PSO-based methods. The authors are currently working towards this goal.

More explicitly, for functions $f_{1}, f_{2}, f_{3}, f_{10}$ and $f_{11}$, SPSO achieves the worst performance against peer schemes. That undesirable behavior is severely conditioned by the topological properties of such problems, where existing local optima can easily misguide the particle swarm to move away from the true global optimum. In this context, FER-PSO seems to be more consistent but it definitely cannot outperform the improved lbest models, which speaks about the efficacy of the proposed methodology. As an illustrative example, Figure 2 unveils the niching behavior induced on the whole swarm by improved ring-based lbest models, where r3PSO-DC was able to discover all global peaks of the Vincent-2D function.
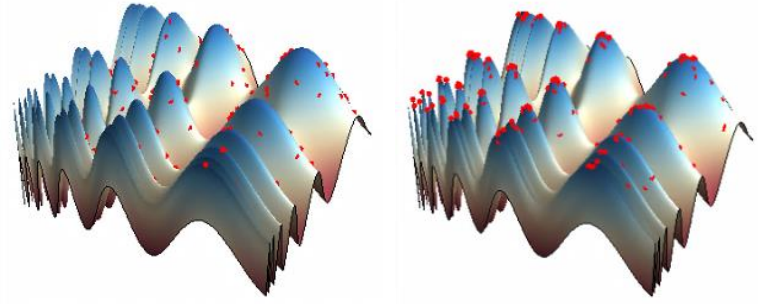

Figure 2. Niching behavior induced by the proposed lbest model over Vincent-2D function a) at initial steps b) at final steps

In a second experiment, we studied the scalability features of the algorithms for two complex benchmarks, i.e., Inverted Shubert $\left(f_{11}\right)$ and Inverted Vincent $\left(f_{12}\right)$ functions. They serve this purpose quite well since they display a similar behavior: the number of local and global optima exponentially increases with the dimensionality of the solution space. For instance, the number of global solutions of Inverted Shubert-3D is 81 , but for a 4D-search space there are 324 global optima (while it has a huge number of suboptimal solutions).

TABLE I. SUCCESS RATES FOR SIMPLER FUNCTIONS

\begin{tabular}{|c|c|c|c|c|c|c|c|c|c|c|c|c|c|}
\hline Id & $s$ & $r$ & $\varepsilon$ & SPSO & FER-PSO & r2PSO & r3PSO & r2hPSO & r3hPSO & r2PSO-DC & r3PSO-DC & r2hPSO-DC & r3hPSO-DC \\
\hline$\overline{f_{1}{ }^{1 \mathrm{D}}}$ & 1 & 0.50 & 0.10 & $24 \%$ & $88 \%$ & $98 \%$ & $100 \%$ & $94 \%$ & $78 \%$ & $100 \%$ & $100 \%$ & $100 \%$ & $100 \%$ \\
\hline$f_{2}{ }^{1 \mathrm{D}}$ & 1 & 0.50 & 0.10 & $22 \%$ & $100 \%$ & $100 \%$ & $96 \%$ & $98 \%$ & $88 \%$ & $100 \%$ & $100 \%$ & $100 \%$ & $100 \%$ \\
\hline$f_{3}{ }^{1 \mathrm{D}}$ & 2 & 0.50 & 5.00 & $40 \%$ & $98 \%$ & $100 \%$ & $96 \%$ & $96 \%$ & $96 \%$ & $100 \%$ & $100 \%$ & $100 \%$ & $100 \%$ \\
\hline$f_{4}{ }^{1 \mathrm{D}}$ & 5 & 0.01 & 0.01 & $100 \%$ & $100 \%$ & $100 \%$ & $100 \%$ & $100 \%$ & $100 \%$ & $100 \%$ & $100 \%$ & $100 \%$ & $100 \%$ \\
\hline$f_{5}^{1 \mathrm{D}}$ & 1 & 0.01 & 0.01 & $100 \%$ & $100 \%$ & $98 \%$ & $100 \%$ & $100 \%$ & $100 \%$ & $100 \%$ & $100 \%$ & $100 \%$ & $100 \%$ \\
\hline$f_{6}{ }^{1 \mathrm{D}}$ & 5 & 0.01 & 0.01 & $100 \%$ & $100 \%$ & $98 \%$ & $98 \%$ & $100 \%$ & $100 \%$ & $100 \%$ & $100 \%$ & $100 \%$ & $100 \%$ \\
\hline$f_{7}^{1 \mathrm{D}}$ & 1 & 0.01 & 0.01 & $100 \%$ & $100 \%$ & $100 \%$ & $100 \%$ & $100 \%$ & $100 \%$ & $100 \%$ & $100 \%$ & $100 \%$ & $100 \%$ \\
\hline$f_{8}^{2 \mathrm{D}}$ & 4 & 0.50 & 0.10 & $100 \%$ & $98 \%$ & $92 \%$ & $74 \%$ & $100 \%$ & $98 \%$ & $100 \%$ & $100 \%$ & $100 \%$ & $100 \%$ \\
\hline$f_{9}^{2 \mathrm{D}}$ & 2 & 0.50 & 0.01 & $100 \%$ & $100 \%$ & $100 \%$ & $100 \%$ & $100 \%$ & $100 \%$ & $100 \%$ & $100 \%$ & $100 \%$ & $100 \%$ \\
\hline$f_{10}^{2 \mathrm{D}}$ & 1 & 0.50 & 0.01 & $50 \%$ & $100 \%$ & $100 \%$ & $100 \%$ & $72 \%$ & $78 \%$ & $100 \%$ & $100 \%$ & $100 \%$ & $100 \%$ \\
\hline$f_{11}^{2 \mathrm{D}}$ & 18 & 0.50 & 0.10 & $49 \%$ & $56 \%$ & $90 \%$ & $98 \%$ & $98 \%$ & $100 \%$ & $100 \%$ & $100 \%$ & $100 \%$ & $100 \%$ \\
\hline$f_{12}^{1 \mathrm{D}}$ & 6 & 0.20 & 0.01 & $84 \%$ & $88 \%$ & $94 \%$ & $86 \%$ & $92 \%$ & $90 \%$ & $100 \%$ & $100 \%$ & $100 \%$ & $100 \%$ \\
\hline
\end{tabular}


TABLE II. PEAK RATIO FOR CHALLENGING FUNCTIONS

\begin{tabular}{|c|ccc|cccccccccc|}
\hline Id & $\boldsymbol{s}$ & $\boldsymbol{r}$ & $\boldsymbol{\varepsilon}$ & SPSO & FER-PSO & r2PSO & r3PSO & r2hPSO & r3hPSO & r2PSO-DC & r3PSO-DC & r2hPSO-DC & r3hPSO-DC \\
\hline \hline$f_{11}{ }^{3 \mathrm{D}}$ & 81 & 0.50 & 0.20 & 0.01 & 0.03 & 0.16 & 0.61 & 0.27 & 0.66 & 0.16 & $\mathbf{0 . 9 2}$ & 0.32 & 0.78 \\
$f_{11}{ }^{4 \mathrm{D}}$ & 324 & 0.50 & 0.20 & 0.00 & 0.00 & 0.00 & 0.25 & 0.00 & 0.14 & 0.00 & $\mathbf{0 . 2 7}$ & 0.00 & 0.14 \\
$f_{12}{ }^{2 \mathrm{D}}$ & 36 & 0.20 & 0.01 & 0.77 & 0.65 & 0.69 & 0.61 & 0.69 & 0.66 & 0.94 & 0.95 & $\mathbf{0 . 9 6}$ & 0.94 \\
$f_{12}{ }^{3 \mathrm{D}}$ & 216 & 0.20 & 0.01 & 0.34 & 0.31 & 0.35 & 0.30 & 0.38 & 0.37 & 0.40 & 0.41 & $\mathbf{0 . 4 8}$ & 0.42 \\
\hline
\end{tabular}

It is obvious that, even with a large enough population size, it was becoming problematic to find all peaks in any run. For this reason, the next experiment only considers the peak ratio attained by each algorithm instead of the success rate. From these simulations, we observed that the improved lbest models notably outperform other methods for all functions, thus finding a higher number of global optima.

In the model implementation, the self-adaptive parametric optimization only got activated at the iteration $t$ if a proper number of objective function evaluations were reached (e.g. $t>M / 4$ ). In this sense, the clearing operator may be more difficult to handle since it could indiscriminately disperse the swarm, hence bringing about serious problems. However, this undesirable effect is actually notably reduced due to the local properties of the clearing operator.

\section{CONCLUSIONS}

Several niching methods have been proposed in the literature, though a large number of them are impractical due to their reliance on additional knowledge about the problem domain. This paper presents a revamped lbest PSO version termed S-PSO-DC with augmented capabilities to face multimodal problems. The differential operator in S-PSO-DC uses knowledge attained by other subpopulations to provoke a controlled perturbation in the lbest search process, exploring new regions of the search space. The heuristic clearing operator in S-PSO-DC is able to preserve the swarm diversity without discarding previously encountered solutions.

Furthermore, S-PSO-DC possesses a self-adaptive scheme to adjust control parameters. In that way, the end user gets a self-adaptive black box framework for solving multimodal optimization problems. S-PSO-DC was tested against 12 wellknown functions of varying difficulty and reported superior performance in all cases against six other niching methods. Future work will concentrate on improving the global scalability of the lbest PSO models, thus enhancing the applicability and robustness of our method.

\section{ACKNOWLEDGEMENT}

This work was supported in the framework of the IT4 Innovations Centre of Excellence project, reg. no. CZ.1.05/1.1.00/02.0070 by operational programme Research \& Development for Innovations funded by the Structural Funds of the European Union and state budget of the Czech Republic.

\section{REFERENCES}

[1] M. G. Epitropakis, V. P. Plagianakos and M. N. Vrahatis, "Finding multiple global optima exploiting differential evolution's niching capability," in 2011 IEEE Symposium on Differential Evolution (SDE)

[2] S. Bird and X. Li, "Adaptively choosing niching parameters in a PSO," in Genetic \& Evolutionary Computation Conference (GECCO), 2006.

[3] Y. Liu, X. Ling, Z. Shi, M. Lv, J. Fang and L. Zhang, "2011 A survey on particle swarm optimization algorithms for multimodal function optimization," Journal of Software, vol. 6, no. 12, pp. 2449-2455, 2011.

[4] E. Dilettoso and N. Salerno, "A self-adaptive niching GA for multimodal optimization of electromagnetic devices," IEEE Transactions on Magnetics, vol. 42, no. 4, pp. 1203-1206, 2006.

[5] S. W. Mahfoud, "Niching methods for genetic algorithms," $\mathrm{PhD}$ Dissertation, University of Illinois at Urbana-Champaign, 1995.

[6] E. Mayr, "Systematics and the origin of species from the viewpoint of a zoologist", Columbia University Press, 1942.

[7] A. Della Cioppa, C. De Stefano and A. Marcelli, "Where are the niches? Dynamic fitness sharing," IEEE Transactions on Evolutionary Computation, vol. 11, no. 4, pp. 453-465, 2007.

[8] S. Das, S. Maity, B.-Y. Qu and P. Suganthan, "Real-parameter evolutionary multimodal optimization - A survey of the state-of-theart," Swarm \& Evolutionary Computation, vol. 1, no. 2, pp. 71-88, 2011.

[9] G. Dick, "Automatic identification of the niche radius using spatiallystructured clearing methods," in IEEE Congress on Evolutionary Computation (CEC), 2010.

[10] X. Li, "Niching without niching parameters: particle swarm optimization using a ring topology," IEEE Transactions on Evolutionary Computation, vol. 14, no. 1, pp. 150-169, 2010.

[11] J. Kennedy and R. Eberhart, "Particle swarm optimization," in IEEE International Conference on Neural Networks, 1995.

[12] M. Clerc and J. Kennedy, "The particle swarm - explosion, stability and convergence in a multidimensional complex space," IEEE Transactions on Evolutionary Computation, vol. 6, no. 1, pp. 58-73, 2002.

[13] D. Parrott and X. Li, "Locating and tracking multiple dynamic optima by a particle swarm model using speciation," IEEE Transactions on Evolutionary Computation, vol. 10, no. 440-458, 2006.

[14] R. Storn and K. Price, "Differential evolution - a simple and efficient heuristic for global optimization over continuous spaces," Journal of Global Optimization, vol. 11, pp. 341-359, 1997.

[15] A. Petrowski, "A clearing procedure as a niching method for genetic algorithms," in IEEE International Conference on Evolutionary Computation, 1996.

[16] D. H. Wolpert and W. G. Macready, "No free lunch theorems for optimization," IEEE Transactions on Evolutionary Computation, vol. 1, no. 1, pp. 67-82, 1997.

[17] O. Olorunda and A. Engelbrecht, "Measuring exploration/exploitation in particle swarms using swarm diversity," in IEEE CEC, 2008.

[18] X. Li, "A multimodal particle swarm optimizer based on fitness Euclidean-distance ratio," in GECCO, 2007. 